\title{
Oxidation State Determination from Chemical Shift Measurements using a Cryogen-Free Microcalorimeter X-Ray Spectrometer on an SEM
} \author{
Ullom $^{3}$ \\ 1. STAR Cryoelectronics Inc., Santa Fe, NM, USA; \\ 2. Los Alamos National Laboratory, Los Alamos, NM USA; \\ 3. National Institute of Standards and Technology, Boulder, CO, USA; \\ 4. H.K.N. Inc., San Jose, CA, USA
}

Robin Cantor ${ }^{1}$, Mark P. Croce ${ }^{2}$, George J. Havrilla ${ }^{2}$, Matthew Carpenter ${ }^{1}$, Kathryn McIntosh ${ }^{2}$, Ad Hall ${ }^{1}$, Stosh A. Kozimor ${ }^{2}$, Hideo Naito ${ }^{4}$, Michael W. Rabin², Dan R. Schmidt ${ }^{3}$, Daniel Swetz ${ }^{3}$ and Joel N.

Cryogenic microcalorimeter X-ray detectors based on superconducting transition edge sensors (TES) offer up to a roughly 60-fold improvement in energy resolution as compared with conventional detectors for energy-dispersive spectrometry. The best energy resolutions demonstrated to date are $2.0 \mathrm{eV}$ full width at half maximum (FWHM) at $1.5 \mathrm{keV}(\mathrm{Al}-\mathrm{K} \alpha)$ and $2.4 \mathrm{eV} \mathrm{FWHM} \mathrm{at} 5.9 \mathrm{keV}(\mathrm{Mn}-\mathrm{K} \alpha)$. The energy resolution of state-of-the-art microcalorimeter detectors rivals the resolution of spectrometers for wavelength-dispersive spectrometry (WDS), yet microcalorimeters offer all the advantages of EDS detectors - ease of use, long-term stability, and the ability to quickly provide qualitative as well as quantitative chemical analysis.

An X-ray microcalorimeter consists of three parts: an absorber that captures the energy of the incident Xray, an extremely sensitive thermometric element to measure the temperature rise of the absorber following an X-ray absorption event, and a support and thermo-isolation structure that determines the rate of heat loss from the microcalorimeter. The thermometric element for a TES microcalorimeter consists of a superconducting thin film operated at its superconducting transition. A TES is particularly attractive for these applications owing to its high sensitivity $\delta \mathrm{E}=d(\log R) / d(\log T)$ and fast response time achievable using a novel electrothermal feedback technique. Normal metal/superconductor bilayers are typically used to fabricate transition edge sensors, since the superconducting critical temperature can easily be tuned to the desired operating temperature (around $0.1 \mathrm{~K}$ ) by adjusting the strength of the proximity-effect coupling, and they can be made with low resistivity to ensure fast thermal response times. Following a photoabsorption event, the sudden temperature rise of the microcalorimeter results in a current pulse through the TES (the height of the pulse being proportional to the incident X-ray energy), which is measured using a low input-impedance superconducting quantum interference device (SQUID) amplifier. The third part of a TES microcalorimeter is the thermal isolation structure, usually a thin membrane upon which the TES bilayer and absorber are fabricated. Typical TES microcalorimeters are fabricated on a micromachined silicon nitride membrane formed by depositing a low-stress, silicon-rich silicon nitride film on a Si wafer and then etching the backside of the wafer down to the membrane, or using a surface micromachining technique to preferentially etch away a sacrificial layer underneath the silicon nitride membrane.

We report the measurement of XRF peak shifts associated with the uranium M $\alpha$ line at nominally 3.171 $\mathrm{keV}$ and the iron $\mathrm{L} \alpha$ and $\mathrm{L} \beta$ lines at 707 and $717 \mathrm{eV}$ that are indicative of the oxidation state of the uranium and iron in the compounds. The oxidation state of an element defines the chemistry and molecular bonding of that element. A high resolution microcalorimeter mounted on a scanning electron microscope has provided a transformative approach to oxidation state measurements. The commercial microcalorimeter 
detector with a resolution of around 5-7 eV was used to detect the X-ray fluorescence emission of the different specimens using an electron beam at $7 \mathrm{kV}$ and beam current of $\sim 55 \mu \mathrm{A}$. This excitation was sufficient to generate M $\alpha$ XRF emission from the uranium specimens with oxidation states of $0,+4$ and +6. In each case the M $\alpha$ XRF line was measured for $500 \mathrm{~s}$. The M $\alpha$ spectral peaks from each specimen were fitted with a Gaussian and the peak centroid of the fit recorded. The peak fit shift measured for the 0 and +4 oxidation states was $\sim 1 \mathrm{eV}$ and for the 0 and +6 oxidation states $\sim 4 \mathrm{eV}$, see Figure 1. Multiple measurements verified the reproducibility of the fits. Two iron compounds, ammonium iron citrate $\left(\mathrm{C}_{6} \mathrm{H}_{8} \mathrm{O}_{7} \mathrm{Fe}^{+3} \mathrm{yNH}_{3}\right)$ and iron nitrate $\left(\mathrm{Fe}\left(\mathrm{NO}_{3}\right)_{3} 9 \mathrm{H}_{2} \mathrm{O}\right)$ were measured. While both iron atoms are in the +3 oxidation state, the peak positions and shapes of the peaks are different see Figure 2. The iron results suggest not only a measure of the oxidation state, but a measure of the coordination environment of the iron. Due to the complex nature of the iron L spectra, more sophisticated modeling of the peak fits will be needed to fully understand the scientific basis for peak positions and shapes. Further peak shifts of the nitrogen in the ammonium and nitrate moieties were also measured, indicating the different chemical environments. These results demonstrate the feasibility of oxidation state measurements using microcalorimeter detectors in an SEM.

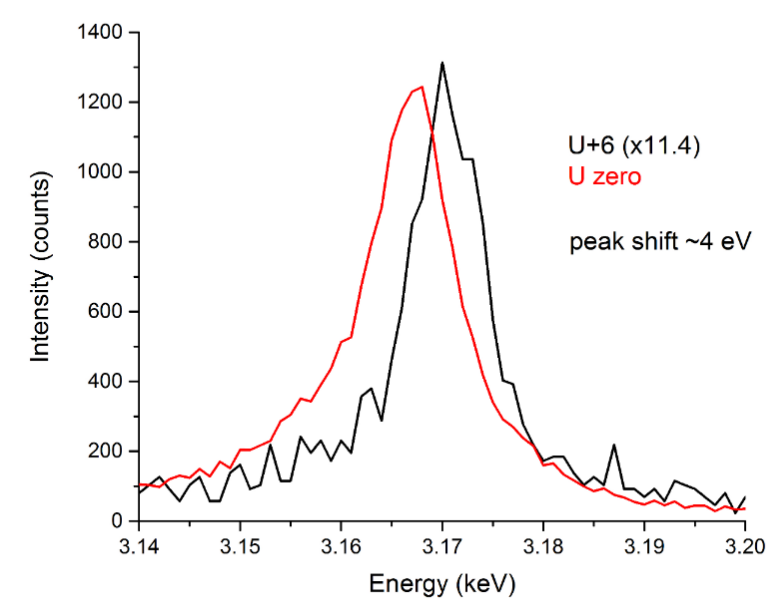

Figure 1. Overlay of $U \mathrm{M} \alpha$ peaks at around $3.17 \mathrm{keV}$ measured for $\mathrm{U} 0$ and $\mathrm{U}+6$ oxidation states using the microcalorimeter in an SEM.

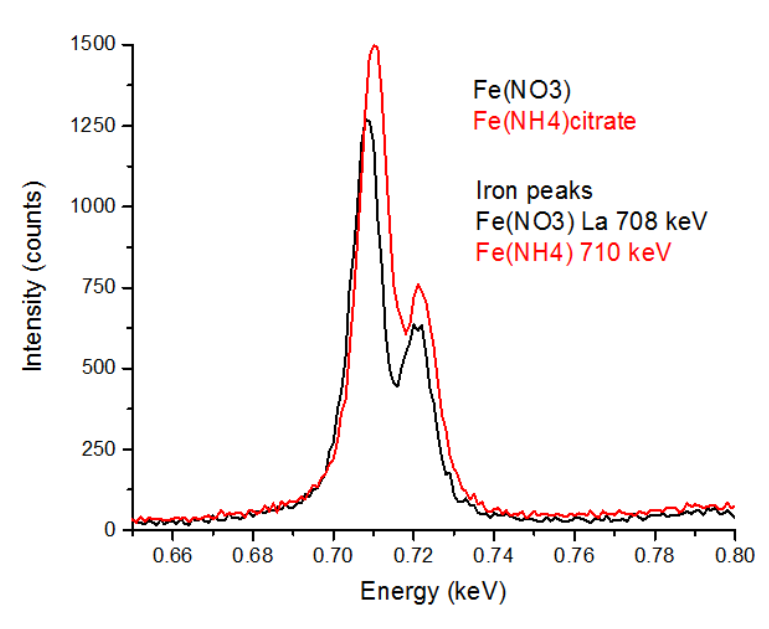

Figure 2. Overlay of Fe L $\alpha$ and $\mathrm{L} \beta$ peaks around $700 \mathrm{eV}$ for two different iron compounds with +3 oxidation state. 